\title{
Reproducing Kernel Function for Sturm-Liouville Problem with Variable Coefficients
}

\author{
Hossam A. Ghany ${ }^{1, *}$, Ashraf Fathallah ${ }^{2}$ \\ ${ }^{1}$ Department of Mathematics, Faculty of Technology, Helwan University, Cairo, Egypt. \\ ${ }^{2}$ Department of Mathematics, Misr International University, Cairo, Egypt.
}

How to cite this paper: Hossam A. Ghany, Ashraf Fathallah. (2021) Reproducing Kernel Function for Sturm-Liouville Problem with Variable Coefficients. Journal of Applied Mathematics and Computation, 5(2), 68-72.

DOI: $10.26855 /$ jamc.2021.06.001

Received: February 22, 2021

Accepted: March 20, 2021

Published: April 12, 2021

*Corresponding author: Hossam A. Ghany, Department of Mathematics, Faculty of Technology, Helwan University, Cairo, Egypt.

Email: h.abdelghany@yahoo.com

\begin{abstract}
This work is committed to find the analytical solution of the versatile Sturm-Liouville equation with variable coefficients by kernelization approach. The introduced generated reproducing kernel Hilbert space (RKHS) structure is subjugated to represent the solution of such problems over the suggested kernel Hilbert space. The advancement of the suggested kernel is built on the matrix structure of the Strum-Liouville operator and the Gram-Schmidt orthogonalization to construct an orthonormal sequences in an inner product Hilbert space. We exhibit the legitimacy of the formalized reproducing kernel Hilbert space to the reckoned Sturm-Liouville differential equation with variable coefficients. Uniform convergence of the approximated solution retaining the recommended scheme is surveyed. The envisaged RHKS, the deployed Sturm-Liouville operator and the analytical solution of the aimed problem are instituted to show the recital of the recommended scheme.
\end{abstract}

\section{Keywords}

Reproducing kernel Hilbert space, Inner product, Sturm-Liouville operator

\section{Introduction}

Recently, some studies have been done in an effort to find the analytical and numerical solutions of Sturm-Liouville differential equation [1]. Analytical solution of some specific types of Sturm-Liouville differential equations with constant coefficients is obtained by several methods in [2] [3]. This paper is devoted to give the reproducing kernel function of the differential equation.

$$
\frac{d}{d x}\left[p(x) \frac{d}{d x}\right] y(x)+\lambda \omega(x) y(x)=q(x) y(x),
$$

where the coefficients $p(x), q(x)$ and $\omega(x)$ are completely monotonic functions, also the eigen functions $u(x)$ satisfy the initial condition $u(0)=0$. The reproducing kernel was first presented in the 1907 through the work of Stanisław Zaremba relating to boundary problems for harmonic functions, concomitantly with that of James Mercer for some integral equations. Reproducing kernel theory has versatile implementations in integral equations, differential equations, probability and statistics [4-6]. Recently, this theory is pragmatic for numerous model problems by many authors. Li and Cui use reproducing kernel theory for finding accurate solution of a special class of nonlinear operator equations in [7]. Geng and Cui in [8], employing an exponential kernel attained an approximate solution for a second order nonlinear system of differential equations. Jiang and Lin applied reproducing kernel theory to get approximate solution of time-fractional telegraph equation in [9].

In [10], Geng constructed a RKHS for attaining a convergent series solution of fourth-order boundary value problems. Arqub et al. in [11], utilized RH theory to find an approximate solution of different sorts of Fredholm integro-differential 
equations. Bushnaq et al. in [12] proposed RH scheme for solving fractional Fredholm integro-differential equations, which results in a uniformly convergent approximate solution with its derivatives. In [13], Geng et al. suggested a modified reproducing kernel method for solving singular perturbation problem. As of late, periodic boundary value problem of two-point second-order mixed integro-differential equation is tackled in [14-18]. A class of second order difference operators with indefinite weights and coupled boundary conditions is treated in [19-20]. The present paper is structured as follows: Section 2 is dedicated to introduce the principle properties of the class of the Hilbert space theory and suggested RKHS. In section 3, we discuss the recommended representation of the Sturm-Liouville operator in equation (1.1). Section 4, is devoted to the main results.

\section{Preliminaries}

A RKHS is related with a kernel that reproduces each function in the considered space as in for any $x$ in the set on which the functions are defined, "estimation of $x$ " can be implemented by taking an inner product with a function determined by the kernel. Such a kernel exists if and just if each functional is continuous. This section introduces the required basic definitions, theorem and reproducing kernel from functional analysis related to Hilbert space [4] [15] [21] [22].

Definition 2.1 Let $X$ be a nonempty set. A function $k: X \times X \rightarrow \mathbb{C}$ is called a reproducing kernel function of the Hilbert space $\mathcal{H}$ if and only if

a) $k(\cdot, t) \in \mathcal{H}$ for all $t \in X$,

b) $\langle\phi, k(\cdot, t)\rangle=\phi(t)$ for all $t \in X$ and $\phi \in \mathcal{H}$. (The reproducing property)

Any Hilbert space $\mathcal{H}$ is called a reproducing kernel Hilbert space, if there exists a reproducing kernel function $k: X \times X \rightarrow \mathbb{C}$ for some nonempty set $X$. Let $A C[a, b]$ denotes the space of absolutely continuous functions on $[a, b]$.

Theorem 2.2 The space $W_{2}^{3}[a, b]:=\left\{u \mid u, u^{\prime}, u^{\prime \prime} \in A C[a, b], u^{(3)} \in L_{2}[a, b]\right\}$ is a reproducing kernel space and its reproducing kernel function is given by

$$
T_{z}(x)=\left\{\begin{array}{ll}
\sum_{i=1}^{6} c_{i}(z) x^{i-1}, & x \leq z \\
\sum_{i=1}^{6} d_{i}(z) x^{i-1} & , z<x
\end{array},\right.
$$

where the inner product and the norm in $W_{2}^{3}[a, b]$ are specified by

For any solution of the form $T_{z} \in W_{2}^{3}[a, b]$, we have

$$
\begin{gathered}
\langle u, v\rangle_{W_{2}^{3}}=\sum_{i=0}^{2} u^{(i)}(a) v^{(i)}(a)+\int_{a}^{b} u^{(3)}(x) v^{(3)}(x) d x, u, v \in W_{2}^{3}[a, b], \\
\|u\|_{W_{2}^{3}}=\sqrt{\langle u, u\rangle_{W_{2}^{3}}} ; u \in W_{2}^{3}[a, b] .
\end{gathered}
$$

$$
<u, T_{z}>_{W_{2}^{3}}=u(a) T_{z}(a)+u^{\prime}(a) T_{z}{ }^{\prime}(a)+u^{\prime \prime}(a) T_{z}{ }^{\prime \prime}(a)+\int_{a}^{b} u^{(3)}(t) T_{z}^{(3)}(t) d t ; u, T_{z} \in W_{2}^{3}[a, b] .
$$

Iterative integration leads to

$$
\begin{array}{r}
<u(t), T_{z}(t)>_{W_{2}^{6}}=u(a)\left[T_{z}(a)-T_{z}^{(5)}(a)\right]+u^{\prime}(a)\left[T_{z}{ }^{\prime}(a)+T_{z}^{(4)}(a)\right]+u^{\prime \prime}(a)\left[T_{z}{ }^{\prime \prime}(a)-T_{z}^{(3)}(a)\right] \\
+u(b) T_{z}^{(5)}(b)-u^{\prime}(b) T_{z}^{(4)}(b)+u^{\prime \prime}(b) T_{z}^{(3)}(b)-\int_{a}^{b} u(t) T_{z}^{(6)}(t) d t .
\end{array}
$$

Since $u, T_{z} \in W_{2}^{3}[a, b]$, it follows that $u(a)=u(b)=T_{z}(a)=T_{z}(b)=0$. Now, the space $W_{2}^{3}[a, b]$ is a reproducing kernel space if for each fixed pointz $\in[a, b]$ and any $u \in W_{2}^{3}[a, b]$, there exists a function $T_{z}$ such that $u(z)=<$ $u(t), T_{Z}(t)>_{W_{2}^{3}}$.

This is attested under the conditions that

$$
T_{z}^{\prime}(a)=-T_{z}^{(4)}(a), T_{z}^{\prime \prime}(a)=T_{z}^{(3)}(a), T_{z}^{(3)}(b)=T_{z}^{(4)}(b)=0 .
$$

Hence, equations (2.2) and (2.3) imply that

$$
\begin{gathered}
<u, T_{z}>_{W_{2}^{3}}=-\int_{a}^{b} u(t) T_{z}^{(6)}(t) d t=u(z), \\
-T_{z}^{(6)}(t)=\delta(t-z) .
\end{gathered}
$$

That is, if $t \neq z$, then $T_{z}^{(6)}(t)=0$.

Equation (2.5) indicates that

$$
\partial^{j} T_{z+}(z)=\partial^{j} T_{z-}(z) ; j=0,1,2,3,4 \text {, and } \partial^{5} T_{z+}(z)-\partial^{5} T_{z-}(z)=-1 .
$$


Thereby, the reproducing kernel function is given by

$$
T_{z}(t)=\left\{\begin{array}{ll}
\sum_{i=1}^{6} c_{i}(z) t^{i-1}, & t \leq z \\
\sum_{i=1}^{6} d_{i}(z) t^{i-1} & , z<t
\end{array} .\right.
$$

As claimed in (2.1), where the coefficients can be determined by using (2.4), (2.6) and $T_{z}(a)=T_{z}(b)=0$. Some numerical examples are deeply considered in [22].

\section{Representation of the Sturm-Liouville operator}

In this section, we provide the matrix representation of the Sturm-Liouville equation (1.1) in the Hilbert space $W_{2}^{3}[0, X]$. Consider the operator $\mathcal{A}: W_{2}^{3}[0, X] \rightarrow W_{2}^{3}[0, X]$ defined by

$$
\mathcal{A} y(x):=P(x) y^{\prime \prime}(x)+P^{\prime}(x) y^{\prime}(x) \text {. }
$$

Thereby, the Sturm-Liouville equation (1.1) can be rewritten in the form

$$
\left\{\begin{array}{c}
\mathcal{A} y(x)=f(x, y), x \in[0, X] \\
y(0)=0
\end{array},\right.
$$

where $f(x, y)=[\lambda \omega(x)-q(x)] y(x)$. It is obvious that the operator $\mathcal{A}: W_{2}^{3}[0, X] \rightarrow W_{2}^{3}[0, X]$ is a bounded linear operator. Setting $\phi_{i}(\mathrm{x})=\mathcal{A}_{x_{i}(x)}$ and $\psi_{i}(\mathrm{x})=\mathcal{A}^{*} \phi_{i}(x)$, where $\mathcal{A}^{*}$ is the adjoint operator of $\mathcal{A}$. Using the Gram-Schmidt orthognalization process [23]of $\left\{\psi_{i}(x)\right\}_{i=1}^{\infty}$, we will obtain the orthogonal $\operatorname{system}\left\{\tilde{\psi}_{i}(x)\right\}_{i=1}^{\infty}$ :

$$
\tilde{\psi}_{i}(x)=\sum_{j=1}^{i} a_{i j} \psi_{i}(x) ; \quad a_{i j}>0, i=1,2, \ldots .
$$

\section{Main Results}

Lemma 4.1 For every dense sequence $\left\{x_{i}\right\}_{i=1}^{\infty}$ on $[0, X]$, the system $\left\{\psi_{i}(x)\right\}_{i=1}^{\infty}$ is a complete system of $W_{2}^{3}[0, X]$ and $\psi_{i}(x)=\left.\mathcal{A}(t) T_{x}(t)\right|_{t=x_{i}}$.

Proof. We have

$$
\begin{gathered}
\psi_{i}(x)=\mathcal{A}^{*}(x) \phi_{i}(x)=<\mathcal{A}^{*}(t) \phi_{i}(t), T_{x}(t)> \\
=<\phi_{i}(t), \mathcal{A}(t) T_{x}(t)>=\left.\mathcal{A}(t) T_{x}(t)\right|_{t=x_{i}}, i=1,2, \ldots
\end{gathered}
$$

Now, let $u(x)=W_{2}^{3}[0, X]$ and $\left\langle u(x), \psi_{i}(x)>=0\right.$ for $i=1,2, \ldots$

Hence, for all $\psi_{i}(x) \in W_{2}^{3}[0, X]$, we have $<u(x), \mathcal{A}^{*}(x) \phi_{i}(x)>=<A(x) u(x), \phi_{i}(x)>=\mathcal{A}\left(x_{i}\right) u\left(x_{i}\right)=0$.

Consequently, $u=0$; since $\left\{x_{i}\right\}_{i=1}^{\infty}$ is a dense sequence on $[0, X]$.

Corollary 4.2 The sequence $\left\{\tilde{\psi}_{i}(x)\right\}_{i=1}^{\infty}$ is the complete basis of $W_{2}^{3}[0, X]$.

Theorem 4.3 For every dense sequence $\left\{x_{i}\right\}_{i=1}^{\infty}$ on $[0, X]$, the solution of the Sturm-Liouville equation (1.1) is given by:

$$
y(x)=\sum_{i=1}^{\infty} \sum_{j=1}^{i} a_{i j} f\left(x_{j}, u\left(x_{i}\right)\right) \tilde{\psi}_{i}(x), a_{i j}>0, i=1,2, \ldots
$$

Proof. Applying the reproducing property

$$
<v(x), \phi_{i}(x)>=v\left(x_{i}\right) \forall v(x) \in W_{2}^{3}[0, X] .
$$

This implies that

$$
\begin{aligned}
y(x) & =\sum_{i=1}^{\infty}<y(x), \tilde{\psi}_{i}(x)>_{W_{2}^{3}[0, X]} \tilde{\psi}_{i}(x) \\
& =\sum_{i=1}^{\infty} \sum_{k=1}^{i} a_{i k}<y(x), \mathcal{A}^{*} T_{x_{k}}(x)>_{W_{2}^{3}[0, X]} \tilde{\psi}_{i}(x) \\
& =\sum_{i=1}^{\infty} \sum_{k=1}^{i} a_{i k}<\mathcal{A} y(x), T_{x_{k}}(x)>_{W_{2}^{3}[0, X]} \tilde{\psi}_{i}(x)
\end{aligned}
$$




$$
\begin{gathered}
=\sum_{i=1}^{\infty} \sum_{k=1}^{i} a_{i k}<f(x, y(x)), T_{x_{k}}(x)>_{W_{2}^{3}[0, X]} \tilde{\psi}_{i}(x) \\
=\sum_{i=1}^{\infty} \sum_{k=1}^{i} a_{i k} f\left(x_{k}, u\left(x_{k}\right)\right) \tilde{\psi}_{i}(x)
\end{gathered}
$$

Corollary 4.4 The approximate solution

$$
y_{N}(x)=\sum_{i=1}^{N} \sum_{j=1}^{i} a_{i j} f\left(x_{j}, u\left(x_{i}\right)\right) \tilde{\psi}_{i}(x), a_{i j}>0, i=1,2, \ldots, N
$$

and its first derivative $y^{\prime}{ }_{N}(x)$ are uniformly convergent on $[0, X]$.

Proof. We have to show that for every solution $y(x)$ in the space $W_{2}^{3}[0, X]$, there exists a certain constant $E>$ 0 such that

$$
\left\|y^{(i)}(x)\right\|_{C[0, X]} \leq E\|y(x)\|_{W_{2}^{3}[0, X]}, i=0,1 ; \text { where }\left\|y^{(i)}(x)\right\|_{C[0, X]}=\max _{x \in[0, X]}|y(x)| .
$$

Since, for every $x \in[0, X], y^{(i)}(x)$ can be written as

$$
y^{(i)}(x)=<y(x), \partial_{x}^{i} T_{x}(x)>_{W_{2}^{3}[0, X]}, \quad i=0,1 .
$$

So,

$$
\begin{gathered}
\left|y^{(i)}(x)\right|=\left|<y(x), \partial_{x}^{i} T_{x}(x)>_{W_{2}^{3}[0, X]}\right| \\
\left|y^{(i)}(x)\right| \leq\|y(x)\|_{W_{2}^{3}[0, X]}\left\|\partial_{x}^{i} T_{x}(x)\right\|_{W_{2}^{3}[0, X]} \leq E_{i}\|y(x)\|_{W_{2}^{3}[0, X]}, i=0,1,
\end{gathered}
$$

where $E_{0}$ and $E_{1}$ are nonzero positive constants. Therefore,

Therefrom, we conclude that

$$
\left\|y^{(i)}(x)\right\|_{C[0, X]} \leq E\|y(x)\|_{W_{2}^{3}[0, X]}, \text { where } E=\max \left\{E_{0}, E_{1}\right\}, i=0,1 .
$$

$$
\begin{gathered}
\left|y_{N}^{(i)}(x)-y^{(i)}(x)\right|=\left|<\left(y_{N}(x)-y(x)\right), \partial_{x}^{i} T_{x}(x)>_{W_{2}^{3}[0, X]}\right| \\
\leq\left\|y_{N}(x)-y(x)\right\|_{W_{2}^{3}[0, X]}\left\|\partial_{x}^{i} T_{x}(x)\right\|_{W_{2}^{3}[0, X]} \\
\leq E\left\|y_{N}(x)-y(x)\right\|_{W_{2}^{3}[0, X]}, i=0,1 .
\end{gathered}
$$

\section{Concluding Remarks}

In this paper, we examine the solution of the Sturm-Liouville differential equation with variable coefficients problem. The reproducing kernel Hilbert space (RKHS) scheme is exploited to figure an answer to the deemed problem. The development of the reproducing kernel based on the matrix representation of the Strum-Liouville operator is introduced. Thence, the Gram-Schmidt orthogonalization process is utilized to deduce a dense set of orthonormal sequences in an inner product Hilbert space. This in turns construct the main core solution of the understudy Strum-Liouville problem. We demonstrate the validity of the proposed RKHS forming the solution of the Sturm-Liouville differential equation with variable coefficients problem. The uniform convergence of the approximate solution and its first derivative employing the proposed method is explored. The results disclose that the proposed analytical RKHS scheme can produce exceptional results in foreseeing the solutions of such versatile Sturm-Liouville problems.

\section{References}

[1] Kikonko, M. (2016). On a Non-Definite Sturm-Liouville Problem in the Two-Turning Point Case-Analysis and Numerical Results. Journal of Applied Mathematics and Physics, 4, 1787-1810. http://dx.doi.org/10.4236/ jamp.2016.49184.

[2] Pryce, J. D. (1993). Numerical Solution of Sturm Liouville Problems. Oxford. Clarendon Press, New York.

[3] Kravchenko, V. V. and Porter, R. M. (2010). Spectral Parameter Power Series for Sturm Liouville Problems. Math. Methods Appl. Sci., 33, 459-468. https://doi.org/10.1002/mma.1205.

[4] Alpay, D. (2003). Reproducing Kernel Spaces and Applications. Birkhauser Verlag, Basel, Switzerland. 
https://doi.org/10.1007/978-3-0348-8077-0.

[5] Berlinet, A. and Thomas-Agnan, C. (2004). Reproducing Kernel Hilbert Spaces in Probability Statistics. Kluwer Academic, Boston, USA.

[6] Vladislav, V. K. (2020). Direct and Inverse Sturm-Liouville Problems: A Method of Solution. Birkhäuser, Cham. https://doi.org/10.1007/978-3-030-47849-0.

[7] Li, C. L. and Cui, M. (2003). The exact solution for solving a class nonlinear operator equations in the reproducing Kernel space. Appl. Math. Comput., 143, 393-399. https://doi.org/10.1016/S0096-3003(02)00370-3.

[8] Geng, F. Z. and Cui, M. (2007). Solving a nonlinear system of second order boundary value problems. J. Math. Anal. Appl., 327, 1167-1181. https://doi.org/10.1016/j.jmaa.2006.05.011.

[9] Jiang, W. and Lin, Y. (2011). Representation of exact solution for the time-fractional telegraph equation in the reproducing Kernel space. Commun. Nonlinear Sci. Numer. Simul., 16, 3639-3645. https://doi.org/10.1016/j.cnsns. 2010.12.019.

[10] Geng, F. (2009). A new reproducing Kernel Hilbert space method for solving nonlinear fourth-order boundary value problems. Appl. Math. Comput., 213, 163-169. https://doi.org/10.1016/j.amc.2009.02.053.

[11] Arqub, O. A., Al-Smadi, M., and Shawagfeh, N. (2013). Solving Fredholm integro-differential equations using reproducing Kernel Hilbert space method. Appl. Math. Comput., 219, 8938-8948. https://doi.org/10.1016/j.amc.2013.03.006.

[12] Bushnaq, S., Momani, S., and Zhou, Y. (2012). A reproducing Kernel Hilbert space method for solving integro-differential equations of fractional order. J. Optim. Theory Appl., 156, 96-105. https://doi.org/10.1007/s10957-012-0207-2.

[13] Geng, F. Z., Qian, S. P., and Cui, M. G. (2015). Improved reproducing Kernel method for singularly perturbed differential-difference equations with boundary layer behavior. Appl. Math. Comput., 252, 58-63. https://doi.org/10.1016/j.amc.2014.11.106.

[14] Boutarfa, B., Akgül, A., and Inc, M. (2017). New approach for the Fornberg-Whitham type equations. J. Comput. Appl. Math., 312, 13-26. https://doi.org/10.1016/j.cam.2015.09.016.

[15] Inc, M., Akgül, A., and Geng, F. (2015). Reproducing Kernel Hilbert space method for solving Bratu's problem. Bulletin of the Malaysian Math. Sci. Soc., 38, 271-287. https://doi.org/10.1007/s40840-014-0018-8.

[16] Akram, G. and Rehman, H. U. (2013). Numerical solution of eighth order boundary value problems in reproducing Kernel space. Numer. Algorithms, 62, 527-540. https://doi.org/10.1007/s11075-012-9608-4.

[17] Geng, F. Z. and Qian, S. P. (2013). Reproducing Kernel method for singularly perturbed turning point problems having twin boundary layers. Appl. Math. Lett., 26, 998-1004. https://doi.org/10.1016/j.aml.2013.05.006.

[18] Kikonko, M. and Mingarelli, A. B. (2013). On Non-Definite Sturm-Liouville Problems with Two Turning Points. Journal of Applied Mathematics and Computing, 219, 9508-9515. http://dx.doi.org/10.1016/j.amc.2013.03.025.

[19] Ma, R., Gao, C., and Lu, Y. (2018). Spectrum Theory of Second-Order Difference Equations with Indefinite Weight. Journal of Spectral Theory, 8, 971-985. https://doi.org/10.4171/JST/219.

[20] Congmin, Y., Yunlan, G., and Kang, S. (2020). Spectrum of a Class of Difference Operators with Indefinite Weights. Journal of Appl. Math. Phys., Vol. 8, No. 4. https://doi.org/10.4236/jamp.2020.84056.

[21] Okutmuştur, B. (2020). A Survey on Hilbert Spaces and Reproducing Kernels. Intechopen. http://dx.doi.org/10.5772/intechopen.91479.

[22] Cui, M. and Lin, Y. (2009). Nonlinear Numerical Analysis in the Reproducing Kernel Space. NY, USA: Nova Science Publishers, Inc.

[23] Akhiezer, N. I. and Glazman, I. M. (1993). Theory of Linear Operators in Hilbert Space. Dover Publications Inc. New York. 